\title{
Editorial: Bioceramics and Bioactive Glasses for Hard Tissue Regeneration
}

\author{
Anuj Kumar ${ }^{1 *}$ and Francesco Baino ${ }^{2}$ \\ ${ }^{1}$ School of Chemical Engineering, Yeungnam University, Gyeongsan, South Korea, ${ }^{2}$ Department of Applied Science and \\ Technology, Institute of Materials Physics and Engineering, Politecnico di Torino, Turin, Italy
}

Keywords: bioceramics, bioactive glasses, hydroxyapatite, calcium phosphates, bone tissue regeneration, additive manufacturing, 3D printing, scaffolds

\section{Editorial on the Research Topic}

Bioceramics and Bioactive Glasses for Hard Tissue Regeneration

Since the 1980s, bioceramics (BCs) and bioactive glasses (BGs) have been used promisingly in orthopedics and dentistry to repair or replace damaged tissues. These biomaterials comprise a broad range of calcium phosphates $(\mathrm{CaPs})$ based on their compositions and $\mathrm{Ca} / \mathrm{P}$ molar ratio [e.g., amorphous calcium phosphates (amorphous-CaP, Ca/P: 1.2-2.2), a-tricalcium phosphate $(\alpha-\mathrm{TCP}, \mathrm{Ca} / \mathrm{P}: 1.5)$, $\beta$-tricalcium phosphate $(\beta-\mathrm{TCP}, \mathrm{Ca} / \mathrm{P}: 1.5)$, hydroxyapatite (HAp, Ca/P: 1.67)], calcium silicates, glasses, and glass-ceramics (Rahaman, 2014). The initial use of these materials as fillers in bone defects has progressively extended to broader therapeutic applications in tissue engineering. Their specific chemical compositions and topographical features have promising effects on cellular response to enhance hard tissue regeneration through cell attachment, migration, proliferation, and differentiation in a three-dimensional (3D) micro-/ nano-environment. Furthermore, appropriate mechanical performance and biocompatibility of these materials are two critical issues for their possible and successful clinical translation.

OPEN ACCESS

Edited by:

Masoud Mozafari,

University of Toronto, Canada

*Correspondence:

Anuj Kumar

anuj.budhera@gmail.com

Specialty section:

This article was submitted to Biomaterials,

a section of the journal

Frontiers in Materials

Received: 11 August 2020

Accepted: 18 August 2020

Published: 04 September 2020

Citation:

Kumar A and Baino F (2020) Editorial: Bioceramics and Bioactive Glasses for

Hard Tissue Regeneration.

Front. Mater. 7:593624.

doi: 10.3389/fmats.2020.593624
Therefore, there is a requirement to provide collective information and future perspectives or research directions on novel ideas and concepts to synthesize and characterize BCs and BGs, with emphasis on their chemical and structural characteristics, design of new compositions of BCs and BGs, their ease of processing with desired surface characteristics or topographies, and their mechanical and cellular responses in regulating cell fate in a $3 \mathrm{D}$ micro-/nano-environment for hard tissue regeneration.

Tissue engineering (i.e., hard tissue regeneration) involves the combination of functional biomaterials and cells, including biomolecules and growth factors. Here, these bioactive materials have the ability to react with physiological fluids for making a firm bonding with living tissue through the formation of apatite layers that lead to an effective interaction with the biological system and attachment of bone tissue with the biomaterial surface (Hench, 1998; Kokubo et al., 2003; Kargozar et al., 2019). Functional biomaterials or scaffolds are highly porous structures with good pore interconnectivity and mechanical performance, and the efficacy of these properties (structure-property relationships) in biomedical applications is dependent on the fabrication technologies used (e.g., electrospinning, freeze-drying, and additive manufacturing methods) in order to achieve promising results with cells and growth factors in a biological environment (Kumar et al., 2017). Therefore, continuous research is expected to consider several interrelated requirements for structural and biological issues while selecting suitable BC- or BG-based biomaterials for fabrication purposes. Furthermore, selection of suitable fabrication techniques is also a major challenge for obtaining scaffolds with desired properties that can mimic natural bone tissue regeneration. Although extensive research studies have already been done based on BCs and/or BGs as well as on their combinations with polymers (composites), several critical issues still remain 
open due to limited understanding of their in vivo behavior; for example, challenges regarding proper cellularization and controlled vascularization of scaffolds need to be resolved before clinical translation (Gerhardt and Boccaccini, 2010). Therefore, the five articles published in this research topic are related to the distinct aspects of the progress of biomaterials based on the use of BCs for hard tissue regeneration, such as $\mathrm{CaP}$ (Baier et al.), siloxane-doped vaterite ( $\mathrm{SiV}$ ) (Obata et al.), HAp (Kumar et al.; Purohit et al.), and HAp/ $\beta$-TCP (d'Arros et al.). In these cases, various fabrication or processing techniques (e.g., data mining, electrospinning, freeze-drying, and additive manufacturing) have been applied to create functional biomaterials for possible use in hard tissue regeneration.

Various types of biomaterials have been used in the repair of hard tissues. Among them, BCs and BGs are suitable biomaterials due to their biodegradability, biocompatibility, osteoinductivity, and osteoconductivity. In this case, sintering of BCs causes the introduction of micropores and also leads to changes in the topographical features on the surface of the BCs. These introduced features have an influence on cellular physiology and bone ingrowth. With this objective, in this Research Topic, Baier et al. used the data mining technique as a new methodological clustering technique to investigate the existence of clusters of micropores and quantify their geometrical properties. Here, three distinctive clusters of micropores were found to be present in $\mathrm{CaP} \mathrm{BC}$ particles based on five main geometrical properties. In this case, around $20 \%$ of the micropores (clusters 1 and 2) possess a Feret's diameter of $50 \mu \mathrm{m}$ or more, and the remaining $80 \%$ of the micropores (cluster 3 ) have a diameter smaller than $20 \mu \mathrm{m}$ or less. This study can also be useful in analyzing other properties, such as mechanical or biological properties, based on the pore clustering effect for bone tissue regeneration. Furthermore, biphasic CPs are largely applied in various clinical situations, and the ratio of biphasic systems is a major parameter modulating their bioactivity due to their different solubilities (Lin et al., 2003). Also, the macro- and microporosity of $\mathrm{CaP}$ BCs serve a fundamental and key role in the osteoinduction process (Eliaz and Metoki, 2017). Low sintering temperature (less than $1,200^{\circ} \mathrm{C}$ ) does not affect the chemical composition and reactivity of the BCs as regards the dissolution or precipitation rates needed for effective bone regeneration (Habibovic et al., 2006). Mechanical mixing of HAp and TCP does not provide the desired BCs, whereas the wet conditions of calcium-deficient HAp followed by sintering convert it into HAp and TCP. Therefore, d'Arros et al. demonstrated the evolution of the crystallographic nature of the initial $\beta$-TCP rhombohedral structure to a microsized needle-like layer analogous to the TCP/apatite (hexagonal) form in a biphasic system of HAp and $\beta$-TCP. This process facilitates the enhancement of the HAp/TCP ratio. Here, high reactivity of biphasic CPs requires a dynamic evolution from a rhombohedral to a hexagonal structure without any change in the chemical entities (unchanged CaP ratio).

Electrospinning technique has been used as a versatile method to produce micro- and nano-fibers to be used in tissue engineering applications. For bone tissue regeneration, Obata et al. used the electrospinning technique to produce fibrous scaffolds composed of polyhydroxybutyrate (PHB) and $\mathrm{SiV}$ with 3D cotton wool-like structures. Here, $\mathrm{SiV}$ releases calcium and silicate ions, which improves the mineralization by osteoblastic cells. Coating of these hydrophobic $\mathrm{PHB} / \mathrm{SiV}$ fibers with imogolite as aluminum silicate nanotubes resulted in a highly hydrophilic surface of the $\mathrm{PHB} / \mathrm{SiV}$ fibrous system, leading to enhanced water penetration into the $3 \mathrm{D}$ scaffold structure $(\sim 2 \mathrm{~s})$. This coating helped cause an improved release of silicate ions from $3 \mathrm{D}$ scaffolds in a cell culture medium and, thereby, an improved attachment and migration of osteoblastic cells (SaOS-2) on the 3D fibrous scaffold. Also, the coating did not affect the mineralization and differentiation of the cells. Furthermore, freeze-drying method has been used to prepare highly porous structures. In this study, HAp was used with graphene oxide (GO) to synthesize HAp/GO nanocomposite systems that were incorporated in a gelatin-alginate (GA) matrix to prepare polymeric nanocomposite scaffolds by using the freeze-drying method. The obtained GA/HAp-GO nanocomposite scaffolds exhibited a porous microstructure and improved compressive mechanical strength as compared to the polymer alone, while reducing swelling (\%) and the rate of biodegradation after the incorporation of the HAp/GO nanocomposite system. Furthermore, the scaffolds showed good in vitro cytocompatibility with MG-63 bone cells. Moreover, an improved calcium deposition and alkaline phosphatase activity were also observed (Purohit et al.).

Additive manufacturing (e.g., 3D printing) methods are considered to be significantly effective in tissue engineering applications and opened new ways in fabricating BC- and BGbased scaffolds for bone tissue regeneration due to their customization ability (e.g., spatial and temporal control) in fabricating patient-specific 3D architectures. HAp is an ideal substitute in repairing of bone tissues due to its crystallographic and compositional similarity to the apatites in hard tissues in the physiological system. But the fabrication of HAp and HAp-based porous scaffolds is a great challenge for appropriate bone tissue regeneration and growth. Despite significant developments, there is still a potential challenge in the design of composition and processing of HAp-based biomaterials, while considering materials, cells, and processing parameters in achieving desired properties mimicking natural hard tissue regeneration. With this objective, Kumar et al. reviewed the additive manufacturing methods for printing HAp and HAp-based biomaterials by involving polymers (e.g., natural or synthetic, or both) or other inorganic materials (e.g., BGs, graphene nanoflakes, wollastonite, and $\beta$-TCP), and also the recent developments in multifunctional HAp-based composite scaffolds for advanced treatments such as hard tissue regeneration and drug delivery applications. Furthermore, in order to better understand the tissue environment and/or developments in the treatments, there is a need of comprehensive, multidisciplinary, and significant research studies for tailoring appropriate properties of complex and strong scaffolds for hard tissue regeneration. Moreover, more research activities on in vivo experiments are required on 
the use of BC- and/or BG-based biomaterials in a realistic biological microenvironment.

The article collection of the research topic Bioceramics and Bioactive Glasses for Hard Tissue Regeneration comprises five articles and is published based on their novel ideas and concepts in the form of original research and review articles through a timely submission and a rigorous review process by the experts in this field. As the guest editors, we would like to thank and appreciate all the authors for their valuable articles and expert reviewers for their valuable time and outstanding efforts, and also the Frontiers editors and publishers who have supported this research topic.

\section{REFERENCES}

Eliaz, N., and Metoki, N. (2017). Calcium phosphate bioceramics: a review of their history, structure, properties, coating technologies and biomedical applications. Materials 10, 334. doi:10.3390/ma10040334

Gerhardt, L.-C., and Boccaccini, A. R. (2010). Bioactive glass and glass-ceramic scaffolds for bone tissue engineering. Acta Biomater. 3 (7), 3867-3910. doi:10. 3390/ma3073867

Habibovic, P., Sees, T. M., van den Doel, M. A., van Blitterswijk, C. A., and de Groot, K. (2006). Osteoinduction by biomaterials-physicochemical and structural influences. J. Biomed. Mater. Res. 77A, 747-762. doi:10.1002/jbm.a.30712

Hench, L. L. (1998). Bioceramics. J. Am. Ceram. Soc. 81, 1705-1728. doi:10.1111/j. 1151-2916.1998.tb02540.x

Kargozar, S., Montazerian, M., Fiume, E., and Baino, F. (2019). Multiple and promising applications of strontium (Sr)-containing bioactive glasses in bone tissue engineering. Front. Bioeng. Biotechnol. 7, 161. doi:10.3389/fbioe.2019. 00161

Kokubo, T., Kim, H.-M., and Kawashita, M. (2003). Novel bioactive materials with different mechanical properties. Biomaterials 24 (13), 2161-2175. doi:10.1016/ S0142-9612(03)00044-9

\section{AUTHOR CONTRIBUTIONS}

All authors listed have made a substantial, direct, and intellectual contribution to the work, and approved it for publication.

\section{FUNDING}

AK gratefully acknowledges the Basic Science Research Program through the National Research Foundation of Korea (NRF) funded by the Ministry of Education, Science and Technology (Grant No. 2017R1D1A3B03036276).

Kumar, A., Rao, K. M., and Han, S. S. (2017). Synthesis of mechanically stiff and bioactive hybrid hydrogels for bone tissue engineering applications. Chem. Eng J. 317, 119-131. doi:10.1016/j.cej.2017.02.065

Lin, S. J., Legeros, R. Z., Rohanizadeh, R., Mijares, D. Q., and Legeros, J. P. (2003). Biphasic calcium phosphate (BCP) bioceramics: preparation and properties. J. Mater. Sci. Mater. Med. 14 (3), 201-209. doi:10.1023/a: 1022872421333

Rahaman, M. N. (2014). "Bioactive ceramics and glasses for tissue engineering," in Tissue engineering using ceramics and polymers. Cambridge, United Kingdom: Woodhead Publishing, 67-114. doi:10.1533/9780857097163.1.67

Conflict of Interest: The authors declare that the research was conducted in the absence of any commercial or financial relationships that could be construed as potential conflict of interest.

Copyright (c) 2020 Kumar and Baino. This is an open-access article distributed under the terms of the Creative Commons Attribution License (CC BY). The use, distribution or reproduction in other forums is permitted, provided the original author(s) and the copyright owner(s) are credited and that the original publication in this journal is cited, in accordance with accepted academic practice. No use, distribution or reproduction is permitted which does not comply with these terms. 